\title{
LENGUA INGLESA APLICADA A LA TRADUCCIÓN. UNA PROPUESTA CURRICULAR ADAPTADA AL ESPACIO EUROPEO DE EDUCACIÓN SUPERIOR Ana Belén Martinez López Universidad de Córdoba
}

Lengua inglesa aplicada a la traducción. Una propuesta curricular adaptada al Espacio Europeo de Educación Superior.

\section{Autor: Richard Clouet.}

Editorial: Editorial Comares, colección interlingua no 86, Granada: 2010.

Reseña: Ana Belén Martínez López (Universidad de Córdoba).

$\mathrm{N}^{\mathrm{o}}$ de páginas: 208.

He aquí una obra necesaria, urgente, y muy pertinente para este momento en el que todos los profesores de Traducción e Interpretación estamos embarcados en la ingente tarea de redefinir nuestras programaciones, de adaptar nuestras metodologías de enseñanza-aprendizaje, de integrarnos plenamente en el "Espacio Europeo de Educación Superior", ya sea para impartir docencia en programas de Grado y/o de Posgrado.

La obra que aquí nos propone Richard Clouet es rigurosa, y en ella se analiza pormenorizadamente tanto el marco jurídico-administrativo en el que se integran las enseñanzas de la Traducción e Interpretación (dentro del nuevo EEES), como el contexto socioeducativo en el que se lleva a cabo esta enseñanza, tomando como referencia el "perfil" de los alumnos que acceden a los Estudios de Traducción e Interpretación en la Universidad española.

El resultado final es una monografía, organizada en tres extensos capítulos, en los que se abordan todos los aspectos que han de ser tenidos en cuenta a la hora de organizar un curso de enseñanza del inglés como lengua $\mathrm{B}$ dentro de los Estudios de Grado en Traducción e Interpretación. A continuación vamos a ir desgranando los contenidos de la obra, capítulo por capítulo, para hacer visible el alcance y la importancia de los temas tratados.

Capítulo 1. Consideraciones generales

En este capítulo, dividido en 6 apartados, se abordan, entre otras cuestiones, las siguientes: 
1. Introducción

2. Naturaleza de los estudios universitarios en el nuevo contexto del Espacio Europeo de Educación Superior

3. El plan de Estudios del Grado en Traducción e Interpretación

4. Los estudios de Traducción y el mundo profesional

5. El perfil de los alumnos del Grado en Traducción e Interpretación

6. Conclusiones del capítulo

Si en el capítulo 1 se nos describe minuciosamente el marco de actuación, el capítulo 2 entra de lleno a considerar, desde una perspectiva teórica, el objeto de estudio.

Éste se titula como sigue: Capítulo 2. Fundamentos teóricos.

Este capítulo, dividido también en 6 apartados, aborda los temas siguientes:

1. Introducción

2. Principales paradigmas lingüísticos

3. Modelos de adquisición de lenguas extranjeras

4. Métodos de enseñanza de lenguas extranjeras

5. Lenguas extranjeras, traducción y comunicación intercultural

6. Conclusiones del capítulo

El capítulo 3, una vez analizados el marco de actuación y los modelos con los que se cuenta para la enseñanza de lenguas extranjeras, se adentra en la definición de una propuesta curricular de enseñanza del Inglés Lengua B en el Grado de Traducción e Interpretación.

El capítulo se titula como sigue: Capítulo 3. Propuesta curricular. En su interior se tratan los siguientes temas:

1. Introducción

2. Inglés para traductores e intérpretes como inglés para fines específicos

3. Objetivos generales de la enseñanza de la lengua B Inglés en un Grado de Traducción e Interpretación

4. Objetivos específicos de la enseñanza de la Lengua B Inglés en un Grado de Traducción e Interpretación

5. Los programas de lengua $B$ Inglés en una Facultad de Traducción e Interpretación

6. Ejemplos de unidades didácticas

7. Civilización y Cultura B Inglés 
8. Metodología docente

Por último, se cierra la monografía con una amplia bibliografía sobre el tema objeto de estudio, organizada por secciones.

No cabe duda que esta obra puede ser entendida como un modelo u obra de referencia para la elaboración de propuestas curriculares en la enseñanza del Inglés Lengua B para Traductores e Intérpretes y, por extensión (realizando los cambios oportunos según la lengua objeto de enseñanza-aprendizaje), para la realización de propuestas curriculares con otras lenguas $\mathrm{B}$ que pudiesen ser objeto de estudio en los Grados de Traducción e Interpretación dentro de la Universidad española.

Sólo nos resta, por último, felicitar y agradecer al autor su generosidad por haberse puesto a plasmar por escrito, de una forma tan exhaustiva, un modelo para el diseño curricular de la enseñanza del inglés (lengua B) en los Estudios de Grado en Traducción e Interpretación, e invitar a los lectores de esta reseña a una lectura atenta de esta obra. 